Programmes d'étude et ressources autorisées 



\section{Programmes d'étude et ressources autorisées}

Savoir les trouver à la bibliothéque de l'Université de l'Alberta

DENIS LACROIX

CGAREAUBRENNAN

UNIVERSITY OF ALBERTA LIBRARY

EDMONTON 


\section{다 (1) (\$) (2)}

Programmes d'étude et ressources autorisées by Denis Lacroix is licensed under a Creative Commons Attribution-NonCommercial-ShareAlike 4.0 International License, except where otherwise noted. 


\section{Contents}

Introduction $\quad 1$

Visite virtuelle de la collection pédagogique de la 2

Bibliothèque Saint-Jean

Part I. I.o A la recherche des programmes d'étude

1.1 Terminologie pédagogique 5

1.2 Point de départ - La bibliothèque de $\quad 6$

l'Université de l'Alberta

1.3 La recherche des programmes d'étude 8

1.4 LearnAlberta et les programmes d'étude $\quad 10$

Exercice 1: Recherche d'un programme d'étude 12

Exercice 2: Recherche d'un programme d'étude 14

1.5 Le nouveau Curriculum sur le nouveau site 15

LearnAlberta.ca

Part II. 2.o A la recherche des ressources autorisées

2.1 Qu'est-ce qu'une ressource autorisée? 19

2.2 Où trouver les ressources autorisées par 20

Alberta Education?

2.3 Comment accéder aux ressources autorisées? 21

2.4 Ce qu'il faut se rappeler pour trouver des 24

ressources autorisées

Exercice 3: La recherche d'une ressources 26

autorisée dans LearnAlberta.ca 
Exercice 4: Les éléments bibliographiques dans

LearnAlberta.ca

Exercice 5: La recherche d'une ressource

autorisée à la bibliothèque

Part III. 3.o Les ressources d'appui

supplémentaires

3.1 Les ressources d'appui dans LearnAlberta.ca

3.2 Ressources d'appui dans les programmes

d'étude

3.3 La base de données actuelle de

LearnAlberta.ca

3.4 Ressources d'appui par la version bêta de LearnAlberta.ca

3.5 Les ressources didactiques en français à la

bibliothèque universitaire

3.6 Accès à la littérature enfantine

Exercice 6: Recherche d'une ressource d'appui par

LearnAlberta.ca

Exercice 7: Encyclopédies

Exercice 8: Ressources d'appui intégrées aux

programmes d'études

Part IV. Questions?

Glossaire 
Ce livre a comme but d'aider les étudiants en éducation du Campus Saint-Jean à trouver des ressources pédagogiques pour leurs cours, leurs stages et leur carrière d'enseignement.

Après avoir lu ce livre et complété les exercices, les étudiants seront capables de

1. Se situer sur le site web de la bibliothèque à l'Université de l'Alberta.

2. Distinguer les sortes d'appuis pédagogiques et leur terminologie

3. Identifier et trouver les programmes d'études et les ressources autorisées

4. Identifier et trouver des ressources numériques 


\section{Visite virtuelle de la \\ collection pédagogique de la Bibliothèque Saint-Jean}

La Bibliothèque Saint-Jean (BSJ) est la bibliothèque où se trouve une grande partie des ressources pédagogiques en français à l'université de l'Alberta. Cependant, d'autres ressources pédagogiques en français se trouvent aussi dans d'autres bibliothèques de notre réseau. Une recherche du catalogue de la bibliothèque vous permettra de savoir où se trouvent les ressources que vous recherchez.

Une visite virtuelle de la BSJ vous est proposée afin de voir comment sont organisées les ressources pédagogiques sur les rayons.

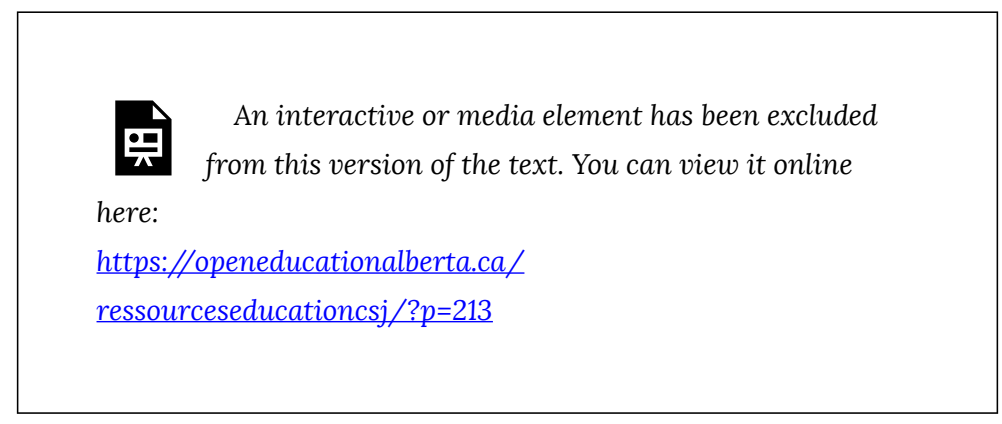

Voici une carte qui représente l'organisation de chaque section de la BSJ. N.B. certaines collections peuvent avoir changées de place. Adressez-vous au personnel de la BSJ pour avoir des précisions. 


\section{PART I}

\section{I.o A LA RECHERCHE DES PROGRAMMES D'ÉTUDE}

Objectifs d'apprentissage

A la suite de ce chapitre, vous serez en mesure de

- d'identifier des programmes d'étude de trois manières différentes

- $\quad$ trouver des programmes d'étude albertains par niveaux scolaires et domaines d'étude 



\section{I.I Terminologie pédagogique}

\section{Mise en situation}

An interactive or media element has been excluded from this version of the text. You can view it online here:

https://openeducationalberta.ca/ ressourceseducationcsj/? $p=29$ 


\section{Le site web de la bibliothèque}

Le site web de la bibliothèque de l'Université de l'Alberta est le point de départ à toute recherche documentaire pédagogique. Tout d'abord, il faut décider quelle sorte de ressource il faut trouver. Dans notre cas, nous sommes à la recherche de programmes d'études albertains. Examinez les trois endroits dans l'image ci-dessous où l'on peut commencer sa recherche de programmes d'étude en cliquant sur le symbole $\mathbf{i}$ pour en savoir plus.

\footnotetext{
An interactive or media element has been excluded

from this version of the text. You can view it online
} here:

https://openeducationalberta.ca/ressourceseducationcsj/?p=5

\section{Le guide par sujet en sciences de l'éducation}

Le guide par sujet ou de recherche Education - Fondements, Psychopédagogie, Méthodologie est le point de référence pour savoir quels outils de recherche utiliser pour trouver des documents pédagogiques. C'est aussi la première manière présentée dans ce manuel pour trouver les programmes d'étude. Examinez, en cliquant

sur le symbole $\mathbf{P}$ pour en savoir plus, les deux endroits dans l'image ci-dessous pour apprendre comment fonctionne le guide et trouver les liens vers les programmes d'étude. 
An interactive or media element has been excluded

from this version of the text. You can view it online

here:

https://openeducationalberta.ca/ressourceseducationcsj/?p=5

Le prochain chapitre vous explique comment trouver des programmes d'étude à partir du site du ministère de l'éducation de l'Alberta. 


\section{I.3 La recherche des programmes d'étude}

\section{Le site de Alberta Education}

La deuxième manière pour retrouver les programmes d'étude consiste à consulter le site web du ministère de l'éducation de l'Alberta. Il suffit de se rendre à l'adresse Internet du ministère ou de LearnAlberta.ca et de suivre les étapes ci-dessous. En cliquant sur le symbole $\mathbf{D}$ dans les images ci-dessous, vous aurez plus d'informations sur le fonctionnement de la page des programmes d'étude du ministère .

1. Naviguer à la section de la page intitulée Ressources pour les enseignants

D An interactive or media element has been excluded from this version of the text. You can view it online here:

https://openeducationalberta.ca/ ressourceseducationcsj/? $\mathrm{p}=31$

2. Sélectionner la matière recherchée et le niveau scolaire en faisant attention à la distinction entre Français (éducation francophone), French Language Arts (programme d'immersion) et French as a second language. Tous les autres programmes sont pareils pour toutes les écoles.

8 | 1.3 La recherche des programmes d'étude 
view it online here:

https://openeducationalberta.ca/

ressourceseducationcsj/?p=31

Les deux prochains chapitres vous présentent une série d'exercices afin mettre en pratique vos habiletés de recherche de programmes d'études. 


\section{I.4 LearnAlberta et les programmes d'étude}

Le site LearnAlberta.ca permet également de trouver des programmes d'étude. Il est peut-être préférable d'accéder aux programmes d'étude de cette manière, car ils intègrent des ressources autorisées et supplémentaires pour approfondir la compréhension et l'enseignement. Tout d'abord il faut se rendre au site LearnAlberta.ca, dont le lien se trouve sur le guide par sujet

en Education (voir l'image ci-dessous et cliquez sur le symbole pour en savoir plus).

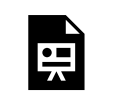
An interactive or media element has been excluded here: https://openeducationalberta.ca/ ressourceseducationcsj/?p=285
}

Dès que vous serez sur la page d'accueil en français de LearnAlberta.ca, veuillez cliquer sur l'onglet Programmes d'étude et suivez les étapes indiquées ci-dessous.

1. Choisissez la langue dans laquelle vous désirez consulter les documents du programme d'études.

2. Sélectionnez un programme parmi les cours obligatoires ou facultatifs en vous servant des deux menus déroulants. 
Voir l'image ci-dessous et cliquez sur le symbole explication visuelle du processus ci-dessus.

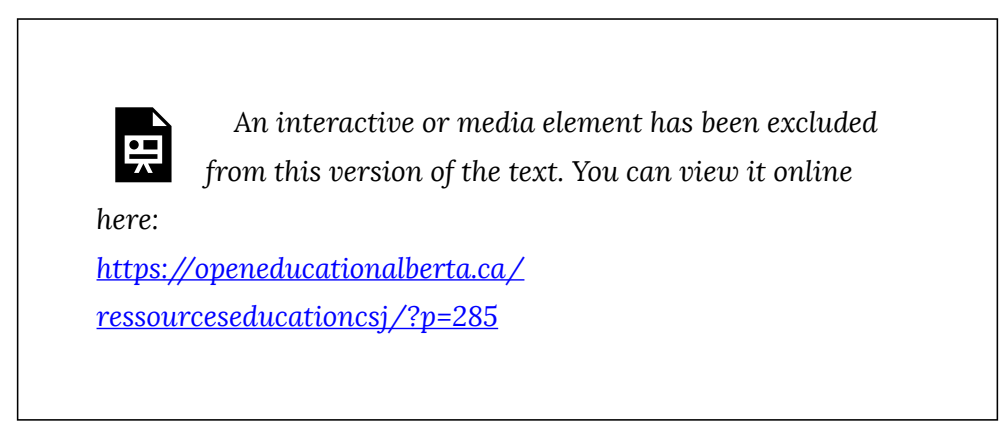

Sélectionnez le programme d'étude qui vous intéresse et utilisez la table des matières pour naviguer d'une section à l'autre. N.B. Une pomme rouge indique qu'il y a une ressource pour l'enseignant associée à cette section. De même l'image d'un cartable indique l'existence d'une ressource pour l'élève. Cliquez sur la pomme ou le cartable pour ouvrir la ressource dans une autre fenêtre. Voir l'exemple ci-dessous et cliquez sur le symbole 1 pour plus d'informations.

An interactive or media element has been excluded
from this version of the text. You can view it online
here:
https://openeducationalberta.ca/
ressourceseducationcsj/? $p=285$




\section{Exercice I: Recherche d'un programme d'étude}

\section{Partie A}

Dans l'image ci-dessous trouvez les 3 endroits par où l'on peut avoir accès au programme d'étude de Mathématiques 1re année.

\footnotetext{
An interactive or media element has been excluded

믓 from this version of the text. You can view it online
} here:

https://openeducationalberta.ca/ressourceseducationcsj/? $p=73$

\section{Partie B}

Dans l'image du site du ministère de l'éducation ci-dessous, trouvez le lien au programme d'étude de Mathématiques 1re année.

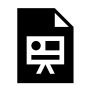

An interactive or media element has been excluded from this version of the text. You can view it online here:

https://openeducationalberta.ca/ressourceseducationcsj/?p=73 


\section{Partie C}

Consultez le programme d'étude de Mathématiques 1re année et répondez aux deux questions suivantes.

An interactive or media element has been excluded
from this version of the text. You can view it online here:

https://openeducationalberta.ca/ressourceseducationcsj/?p=73 


\section{Exercice 2: Recherche d'un programme d'étude}

Pour répondre à la question suivante, veuillez vous référer à la liste des programmes d'étude du ministère de l'éducation de l'Alberta.

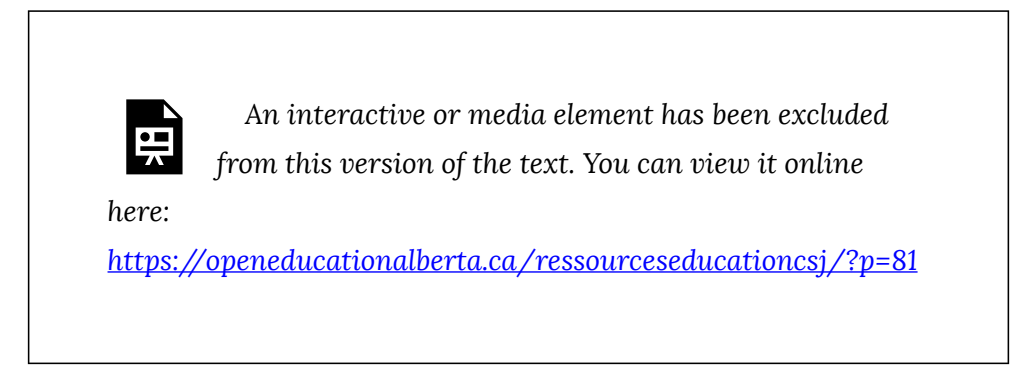

Le prochain chapitre vous présente la version bêta du nouveau site de LearnAlberta.ca, ainsi que le nouveau curriculum. 


\section{I.5 Le nouveau Curriculum sur le nouveau site LearnAlberta.ca}

Un nouveau curriculum pour la maternelle à la 12e année est en voie d'élaboration selon le Cadre directeur pour la conception et l'élaboration du curriculum provincial de la maternelle à la 12e année. Veuillez consulter le site du ministère de l'éducation pour en savoir plus sur l'élaboration du nouveau curriculum. Pour trouver le nouveau curriculum sur la version bêta du nouveau site de LearnAlberta.ca, veuillez suivre les étapes suivantes.

1. Rendez vous à la version bêta du site de LearnAlberta.ca

2. Sélectionnez le curriculum actuel (M à 12) ou futur ( $M$ à 4), ou les deux si vous souhaitez les comparer

3. Choisissez une ou plusieurs matières. N.B. le choix de plusieurs matières permet d'en comparer les objectifs d'apprentissage

\footnotetext{
国 An interactive or media element has been excluded from this version of the text. You can view it online here: https://openeducationalberta.ca/ressourceseducationcsj/?p=84
} 


\section{Exercice pratique:}

A l'aide de la version bêta de LearnAlberta.ca, comparez le programme d'étude actuel et futur pour Maths 1re année.

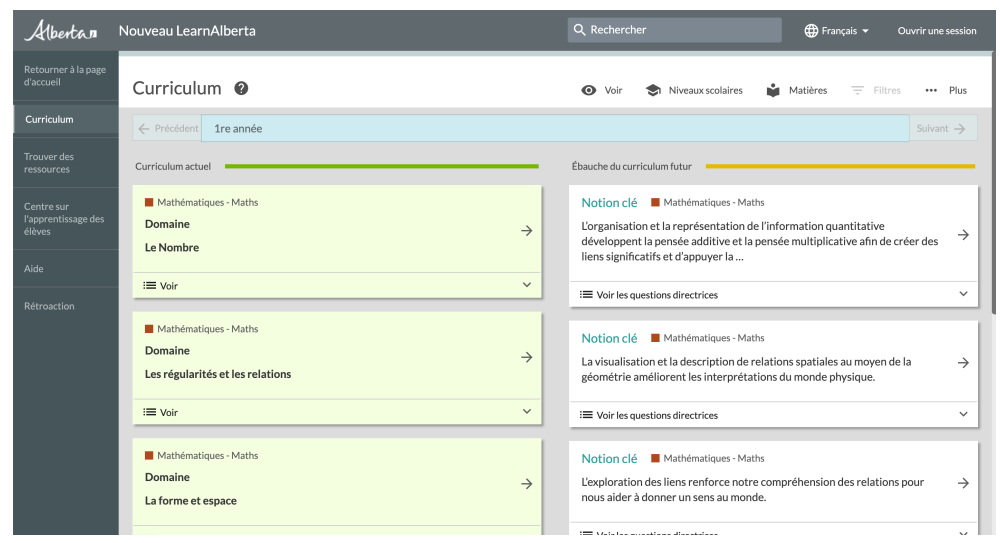

La prochaine partie explique comment trouver des ressources autorisées afin d'aider les élèves à atteindre les objectifs des programmes d'étude. 


\section{PART II}

\section{2.o A LA RECHERCHE DES RESSOURCES AUTORISÉES}

Objectifs d'apprentissage

A la suite des prochains chapitres, vous serez en mesure de

- définir ce qu'est une ressource autorisée

- identifier les manuels utilisés en salles de classe

- $\quad$ trouver d'autres ressources autorisées complémentaires

- $\quad$ situer les ressources autorisées dans les collections de la bibliothèque de l'Université de l'Alberta 



\section{I Qu'est-ce qu'une ressource autorisée?}

A l'aide des choix multiples suivants, sélectionnez la (les) caractéristique(s) d'une ressource pédagogique autorisée.

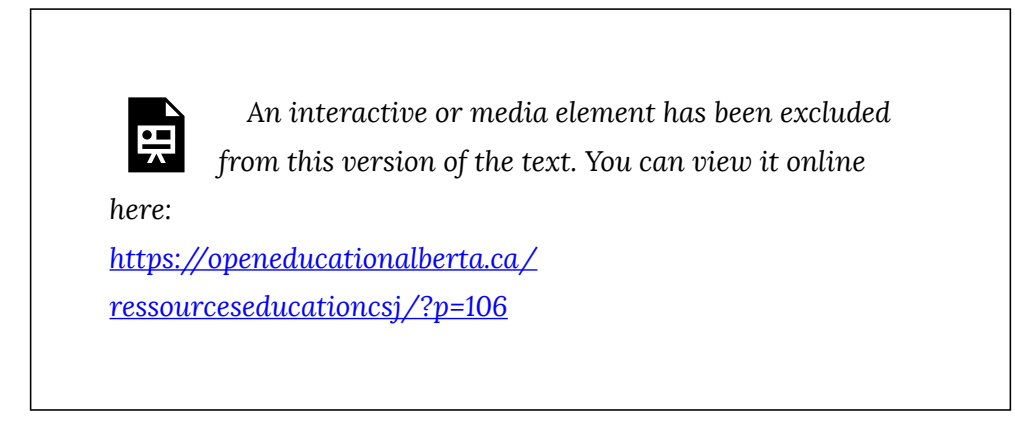

Il y a 3 sortes de ressources autorisées:

1. Les ressources de base: ce sont des ressources destinées aux élèves qui répondent à toutes les attentes des programmes d'études, voir Voix et Visions: une histoire du Canada

2. Les ressources de l'enseignant: ce sont les ressources destinées aux enseignants, certaines sont connues sous le nom de guide d'enseignement. Voir par ex. Liens Mathématiques 7 (guide).

3. Les ressources d'appui: ce sont des ressources autorisées supplémentaires pour aider les élèves à atteindre certains objectifs des programmes d'étude, voir par ex. Les francophones de l'Alberta. 


\subsection{Où trouver les ressources}

\section{autorisées par Alberta Education?}

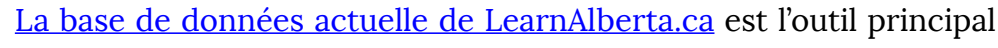
par lequel on trouve des ressources autorisées par Alberta Education. Explorez la base de données actuelle de LearnAlberta.ca en cliquant sur le symbole $\mathbf{D}$ dans l'image ci-dessous. Commencez votre exploration avec le choix de langues du site LearnAlberta.ca situé en haut à gauche de l'écran.

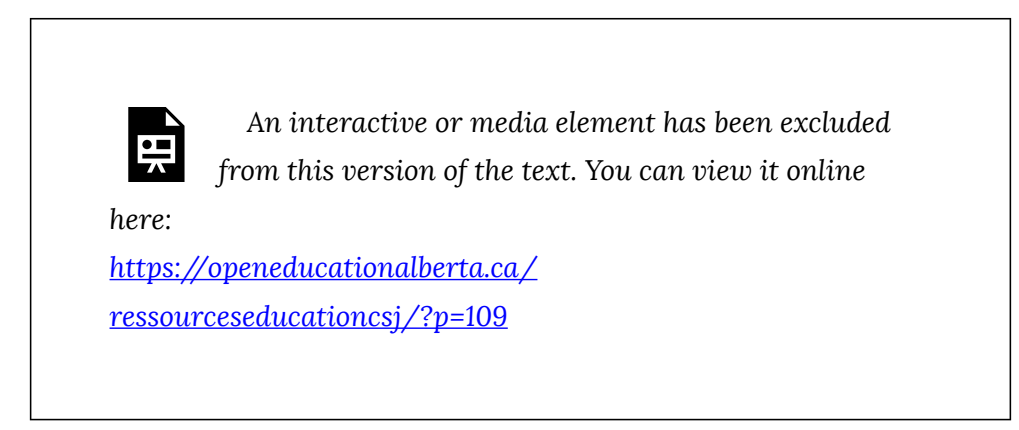




\subsection{Comment accéder aux ressources autorisées?}

Il y a 3 étapes à suivre pour découvrir où se trouve une ressources autorisée identifiée par le site de LearnAlberta.ca. Voir l'explication des étapes ci-dessous, ainsi que dans le guide de la bibliothèque pour EduM.

1. Une fois que vous aurez choisi une ressource, notez le titre, l'ISBN, et l'auteur. Il y a souvent plus qu'une ressource de base par sujet, par niveau scolaire qui sont autorisées. C'est à la discretion de l'enseignant ou de l'école à choisir quel titre utiliser.

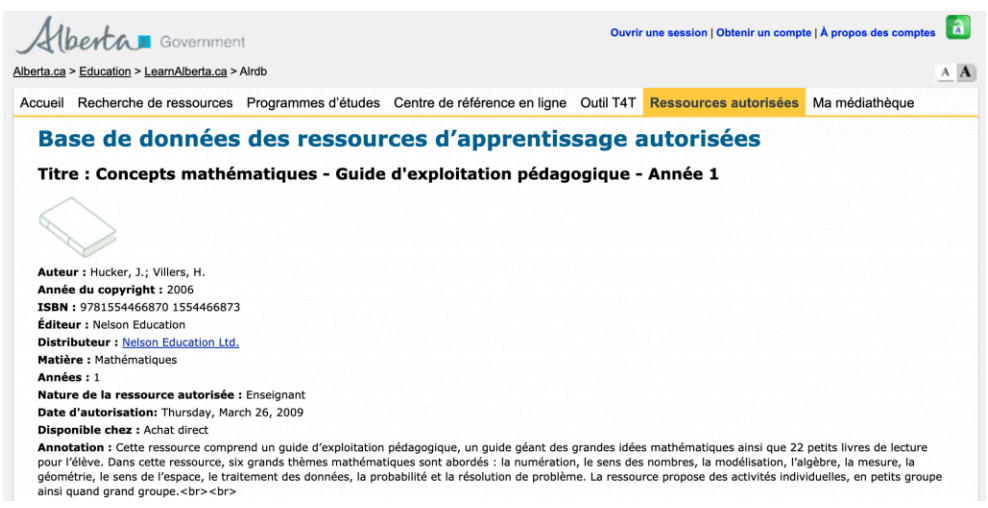

2. Effectuez une recherche dans le catalogue de la bibliothèque pour cette ressource. Une recherche par ISBN est parfois plus efficace. Voir les deux manières d'effectuer une recherche de la même ressources dans le catalogue. Limage de gauche présente une recherche par ISBN dans la boîte de recherche de la bibliothèque situé sur la page d'accueil de la bibliothèque. La manière de droite est une recherche avancée, dont le lien se trouve sur la page d'accueil de la bibliothèque à droite du bouton Recherche. 


\section{Rechercher dans la Bibliothèque}

\section{0}


3. Par exemple, la cote de la ressource, Concepts mathématiques, indiquée dans la notice ci-dessous permet de situer la ressource sur les rayons de la bibliothèque dans la section Curriculum. Veuillez cliquer sur le symbole dans l'image ci-dessous pour en savoir plus sur les différentes parties d'une notice bibliographique.

An interactive or media element has been excluded 믓 from this version of the text. You can view it online here:

https://openeducationalberta.ca/ressourceseducationcsj/?p=131 


\subsection{Ce qu'il faut se rappeler pour trouver des ressources autorisées}

Le guide de recherche en Education de la bibliothèque de l'Université de l'Alberta que vous trouverez en cliquant sur l'onglet Guides par sujet, Bibliothèque Saint-Jean, BSJ Guides et le titre du guide Education - Fondements, Psychopédagogie, Méthodologie. Voir le parcours visuel ci-dessous.

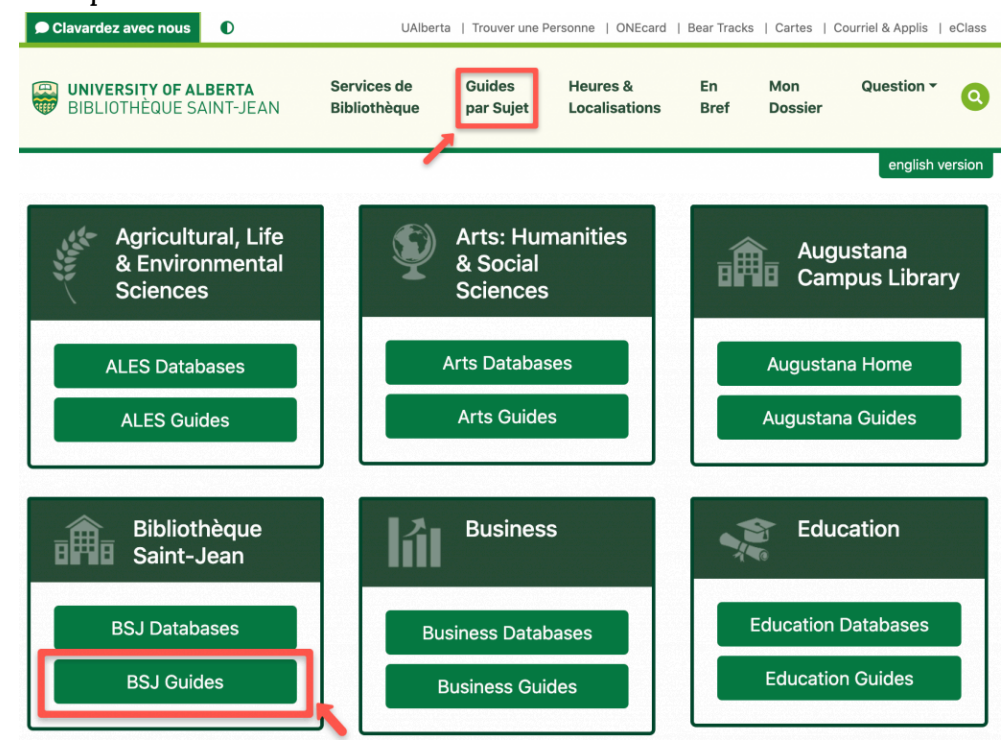

L'image ci-dessous du guide de recherche en Education montre, à l'aide du symbole $\mathbf{1}$, deux endroits où l'on peut trouver des liens aux ressources autorisées, soit sur la page d'accueil avec le lien à la base de données des ressources autorisées par Alberta Education, soit par listes partielles sur les pages d'enseignement des matières. 
An interactive or media element has been excluded

圆 from this version of the text. You can view it online

here:

https://openeducationalberta.ca/

$\underline{\text { ressourceseducationcsj/?p }=160}$

N.B. seule la base de données des ressources autorisées par Alberta Education donne la liste complète des ressources autorisées par le ministère. Afin de trouver les ressources mêmes, il suffit de les rechercher dans le catalogue de la bibliothèque, voir les étapes à suivre dans le guide de recherche sous l'onglet EduM.

Limage ci-dessous du guide de recherche en Education montre, à l'aide du symbole $\mathbf{D}$, comment avoir accès à une liste partielle de ressources autorisées par matière enseignée et niveaux scolaires en cliquant sur la flèche du menu déroulant et en choisissant le lien intitulé ressources autorisées, voir par exemple une liste partielle des ressources autorisées pour l'enseignement des mathématiques.

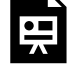

An interactive or media element has been excluded from this version of the text. You can view it online

here:

https://openeducationalberta.ca/

ressourceseducationcsj/?p=160 


\section{Exercice 3: La recherche d'une ressources autorisée dans LearnAlberta.ca}

Dans cet exercice il vous sera demandé de consulter la base de données des ressources autorisées par Alberta Education, intitulé LearnAlberta.ca, afin de trouver un manuel de l'élève, c'est-à-dire une ressource de base, intitulé Théo et Raphaëlle (cahier d'activités) pour l'enseignement du français (Immersion) au niveau 3e année. N.B. référez-vous au chapitre 1.3 pour savoir comment identifier le programme d'immersion d'enseignement du français.

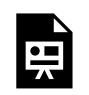
An interactive or media element has been excluded here:

https://openeducationalberta.ca/ressourceseducationcsj/?p=173
} 


\section{Exercice 4: Les éléments bibliographiques dans LearnAlberta.ca}

La notice pour le cahier d'activités de Théo et Raphaëlle que vous avez trouvée dans l'exercice précédent contient des informations bibliographiques importantes afin de le trouver à la bibliothèque. Veuillez vous servir de l'image ci-dessous afin d'indiquer quels trois éléments sont les plus importants afin de trouver le livre à la bibliothèque.

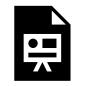

An interactive or media element has been excluded

here:

https://openeducationalberta.ca/ressourceseducationcsj/?p=175 


\section{Exercice 5: La recherche d'une} ressource autorisée à la

\section{bibliothèque}

A l'aide de la recherche avancée du catalogue de la bibliothèque, effectuez une recherche du cahier d'activités Théo et Raphaëlle identifié dans les exercices précédents et répondez aux questions suivantes.

\footnotetext{
An interactive or media element has been excluded 园 from this version of the text. You can view it online here:

https://openeducationalberta.ca/ $\underline{\text { ressourceseducationcsj/? } p=182}$
} 


\section{PART III \\ 3.0 LES RESSOURCES D'APPUI SUPPLÉMENTAIRES}

Objectifs d'apprentissage

A la suite des prochains chapitres, vous serez en mesure de

- découvrir des ressources d'appui par LearnAlberta.ca

- $\quad$ identifier des ressources d'appui par le catalogue de la bibliothèque ou les guides par sujet 



\section{I Les ressources d'appui dans LearnAlberta.ca}

Il y a 3 manières de trouver les ressources d'appui supplémentaires sélectionnées par Alberta Education:

1. Ressources d'appui qui accompagnent les programmes d'étude

2. La base de données actuelle de LearnAlberta.ca

3. La version bêta de LearnAlberta.ca

Explorez les 3 manières dans les prochains chapitres. 


\subsection{Ressources d'appui dans les programmes d'étude}

Les programmes d'étude présentent quelques ressources d'appui pour soutenir les objectifs. Il suffit de consulter le programme d'étude sur le site du ministère d'éducation et de sélectionner l'onglet de gauche intitulé Ressources d'appui. L'exemple ci-dessous présentent quelques ressources d'appui pour Mathématiques (M à 6).

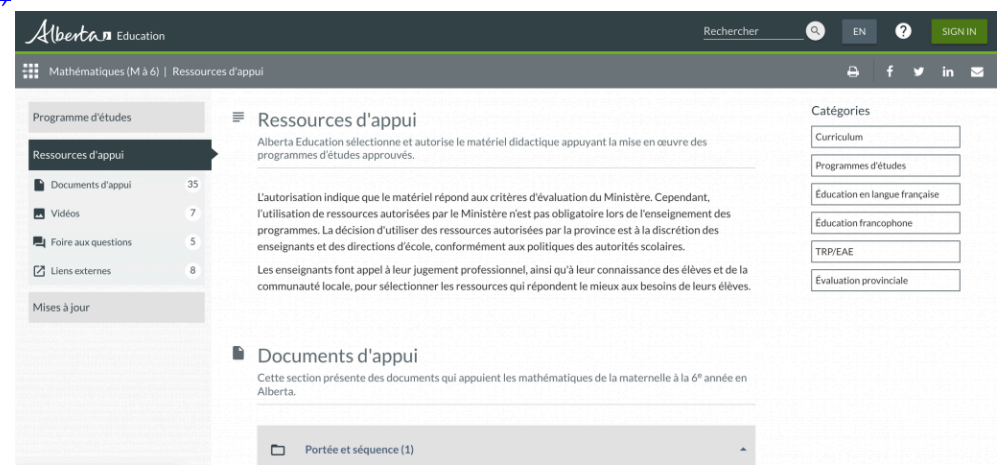




\subsection{La base de données actuelle de LearnAlberta.ca}

La base de données actuelle de LearnAlberta.ca offre la possibilité d'effectuer une recherche parmi toutes les ressources d'appui sélectionnées par le ministère. L'image ci-dessous présente la base de données de ressources d'appui accessible à partir du site LearnAlberta.ca et de l'onglet Recherche de ressources.

N.B. Certaines ressources électroniques requiert une identification préalable auprès de la base de données de LearnAlberta. Veuillez vous identifier en cliquant sur le lien Ouvrir une session situé en haut à droite de l'écran. L'identifiant et le mot de passe sont disponibles aux étudiants et au personnel en sciences de l'éducation de l'université de l'Alberta en contactant la bibliothèque.

\footnotetext{
An interactive or media element has been excluded

읏 from this version of the text. You can view it online
}

here:

https://openeducationalberta.ca/

ressourceseducationcsj/?p=193 
L'onglet intitulé Centre de référence en ligne offre un accès à certaines ressources de référence, telles que des encyclopédies et des dictionnaires, utiles pour l'enseignement au niveau primaire et secondaire en Alberta. Certaines ressources requiert l'ouverture d'une session dans LearnAlberta.ca à l'aide de l'identifiant et du mot de passe. N.B. L'encyclopédie Universalis junior et Universalis senior sont également accessibles par la bibliothèque de l'Université de l'Alberta.

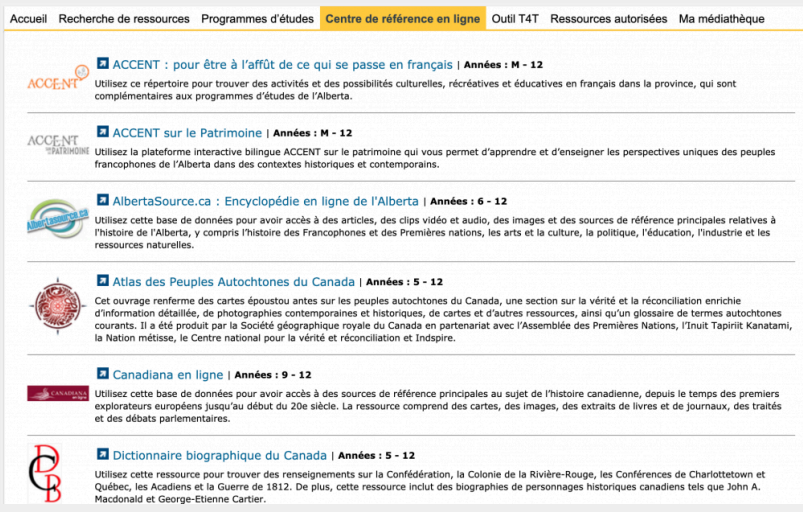

L'onglet Outil T4T de LearnAlberta.ca, accessible seulement après une identification préalable, regroupe des manuels d'élèves, ainsi que des guides pédagogiques. Seuls les manuels sont disponibles à l'aide de l'identifiant mentionné ci-dessus. Par contre, les écoles de la province ont un autre identifiant qui permet un accès plus généralisé. 
Accueil Recherche de ressources Programmes d'études Centre de référence en ligne Outil T4T Ressources autorisées Ma médiathèque

\section{L'outil T4T est maintenant offert sur le site LearnAlberta.ca}

L'outil T4T offre du matériel didactique et pédagogique sécurisé, élaboré par Alberta Education à l'intention des élèves et des enseignants de cette province.

Ce matériel d'apprentissage distribué, élaboré par Distributed Learning (Alberta Education), inclut des ressources pour élèves et enseignants ainsi que des clés de correction. Les enseignants peuvent utiliser ce matériel pour élaborer leurs cours.

Pour commencer,

choisissez une année et une matière.

\begin{tabular}{|l|}
\hline Année $>1^{\text {re année }}$ \\
\hline Matière $>$ choisir une matière \\
\hline
\end{tabular}

\section{Note à l'intention des enseignants}

vous avez ouvert une session dans un compte de rautorité scolaire. Vous ne pourrez consulter que les ressources pour éléves.

vous devez ouvrir une session dans un compte personnel de l'enselgnant afin d'accéder aux ressources pour enselgnants. 


\subsection{Ressources d'appui par la version bêta de LearnAlberta.ca}

A partir de la version bêta de LearnAlberta.ca, cliquez sur la boîte intitulé Trouver des ressources pour accéder à la base de données de ressources (voir l'image de la boîte ci-dessous).

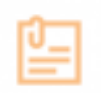

\section{Trouver des ressources}

\section{Rechercher des milliers de ressources éducatives \\ de LearnAlberta pour les enseignants, les élèves et les parents.}

- Suivez les choix proposés par le moteur de recherche (voir l'image ci-dessous) afin de trouver des ressources appropriées, c'est-à-dire

- choisir les niveaux scolaires

- choisir des matières

- entrer un mot-clé (facultatif)

- cliquer sur le bouton Rechercher 


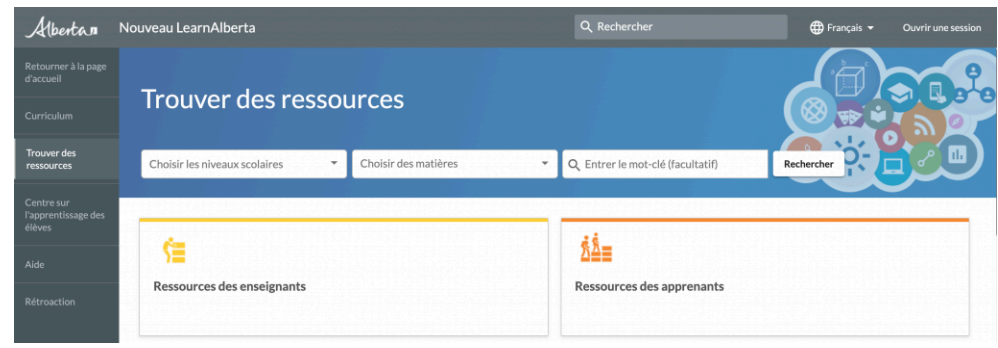

Une option alternative, visible dans l'image ci-dessus, consiste à parcourir la collection de titres en cliquant sur les boîtes intitulées Ressources pour enseignants ou Ressources pour apprenants et en affinant les résultats par la suite. L'image ci-dessous montre les choix proposés pour affiner une recherche, soit par niveaux scolaires, par matières, par public cible, par langues, par formats de médias ou par type de ressources d'apprentissage.

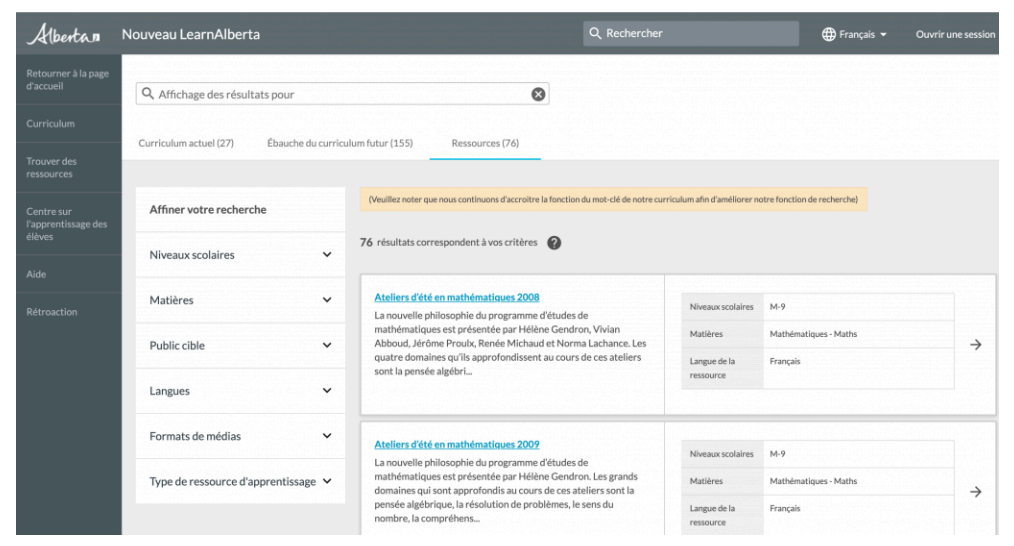




\subsection{Les ressources didactiques en français à la bibliothèque universitaire}

La plupart des ressources publiées récemment et utilisées de manière régulière se trouve à la Bibliothèque Saint-Jean. Cependant, plusieurs ressources didactiques en français se trouvent dans les collections d'autres bibliothèques de l'Université de l'Alberta, dont l'entrepôt RCRF au campus sud. Il est donc important de toujours effectuer une recherche dans le catalogue de la bibliothèque pour avoir une idée précise où est situé ce que l'on cherche.

La boîte de recherche de la page d'accueil de la bibliothèque de l'Université de l'Alberta vous est idéal pour une simple recherche par titre, auteur ou ISBN. Voir l'exemple ci-dessous d'une recherche du titre Sciences Contact 1.

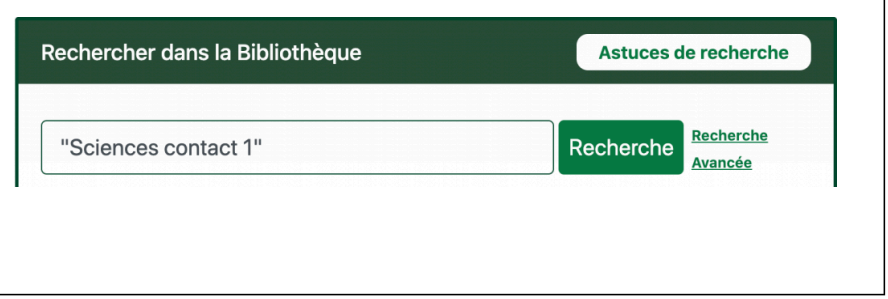

La recherche avancée, dont le lien est visible dans 
l'image ci-dessus à droite du bouton Recherche, permet d'effectuer une recherche plus pointue en précisant le titre ou l'auteur d'un document. Cependant, elle sert aussi à identifier des ressources par vedettes matières et mots-clés. Voici un exemple de recherche avancée cidessous par vedettes matières afin de trouver des manuels scolaires de sciences en français. N.B. il est possible d'ajouter les mots-clés primaire ou secondaire dans le champs All Fields pour limiter les résultats.

\section{Advanced Search}

Find items that match all $\vee$ of

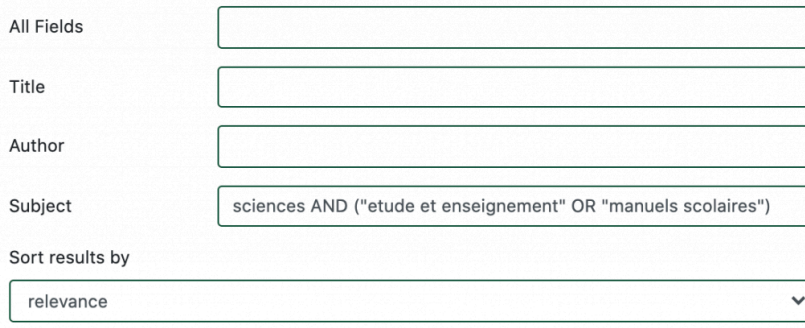




\subsection{Accès à la littérature enfantine}

Le guide de ressources en littérature jeunesse de la bibliothèque de l'Université de l'Alberta permet de voir les différentes options d'accès à la littérature enfantine. Le lien au guide se trouve sous l'onglet l'enseignement du français dans le guide Éducation Fondements, Psychopédagogie, Méthodologie.

1. La première option consiste à effectuer une recherche avancée du catalogue de la bibliothèque en utilisant la vedette matière romans jeunesse et un mot-clé qui décrit le thème qui nous intéresse, par ex. amitié. Veuillez noter que la plupart des livres pour enfants trouvés par le catalogue seront en format imprimé. Voir les résultats de la recherche ci-dessous.

\section{Advanced Search}

Find items that match all $\checkmark$ of

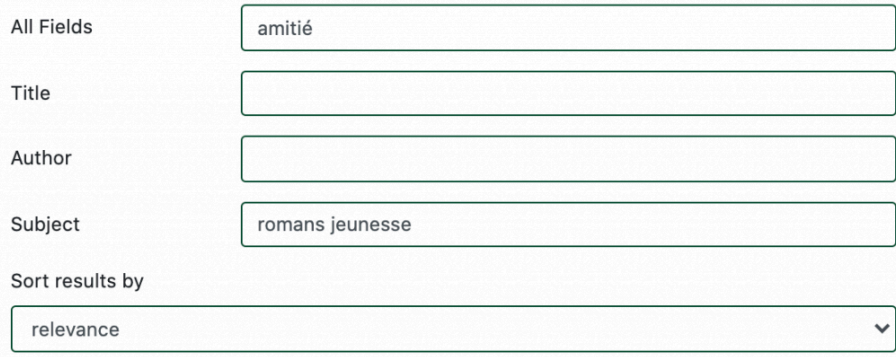

2. La deuxième option consiste à consulter une base de données qui permet une recherche thématique et nivelée selon l'âge des lecteurs parmi les livres publiés. Voici deux bases de données (Mémento et CLCD) qui facilitent l'identification de titres pertinents qu'il sera possible par la suite d'obtenir à la bibliothèque de l'Université de l'Alberta. 
Rechercher dans :

\section{Memento}

Public

Recherche rapide

Recherche avancée

Recherche ISBN

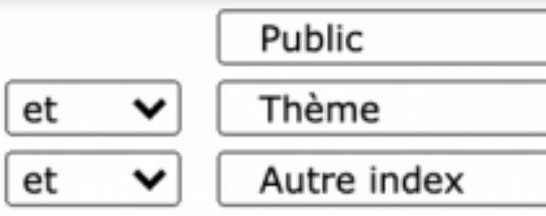

$\boxminus$ Fermer le filtre $\square$ Gard Disponibilité :

$\checkmark$ Dispon Epuisé A para Manqu

Famille :

$\checkmark$ Livres
Cartes
Autres m

$\square$

Périmètre européen Périmètre canadien 
Children's

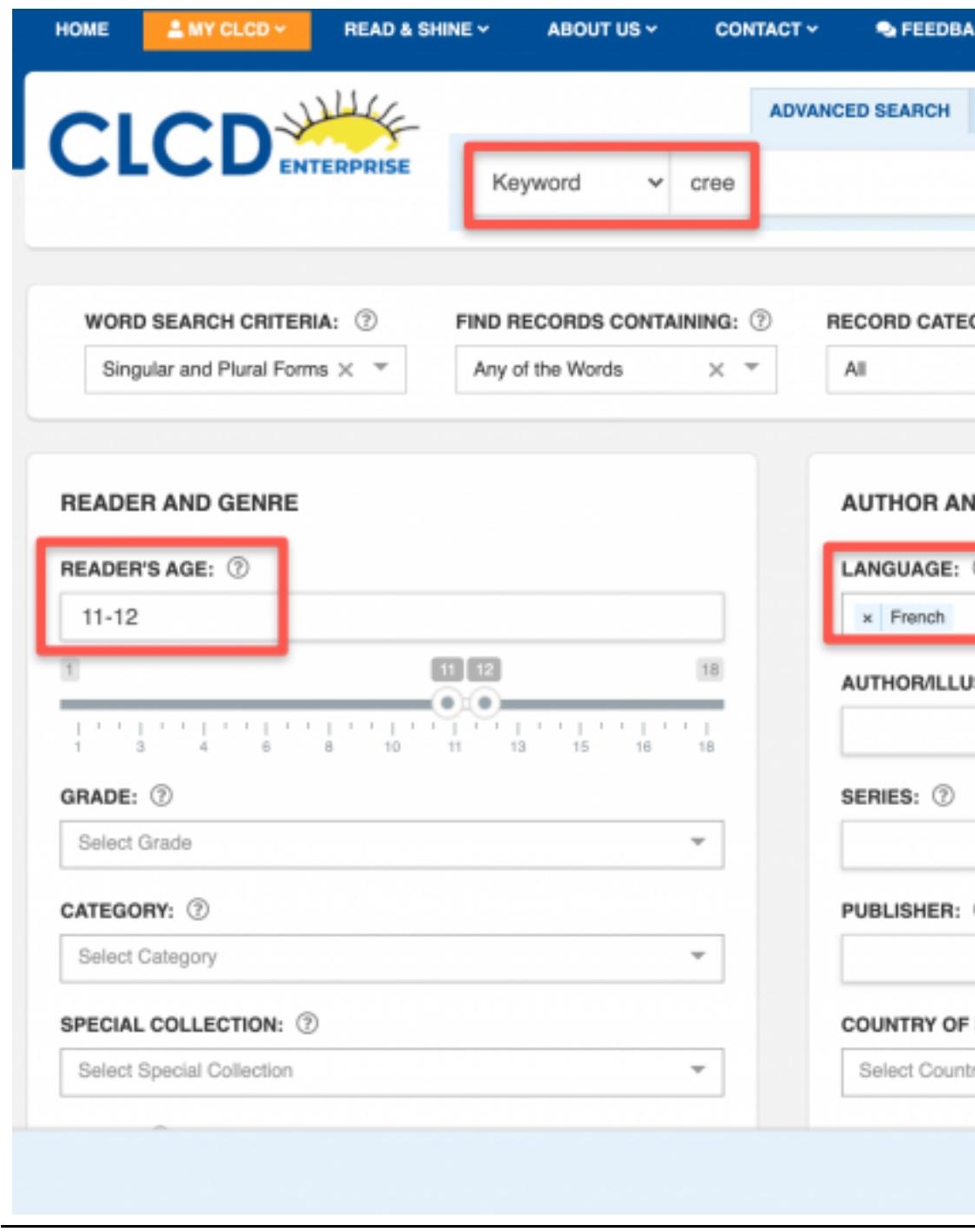

3. Une autre option propose de consulter des bases de données de livres électroniques afin de trouver de la littérature jeunesse en ligne. Il existe quelques bibliothèques numériques à consulter.

42 | 3.6 Accès à la littérature enfantine 
1. dèsLibris

2. Bibliothèque des Amériques

3. Cantook

4. OpenLibrary 


\section{Exercice 6: Recherche d'une ressource d'appui par LearnAlberta.ca}

Servez-vous de la Recherche de ressources prévue sur LearnAlberta.ca afin de trouver le titre d'une vidéo pour les élèves de 12e année, français langue première, qui traite de l'éducation francophone en Alberta. Identifiez le titre de la vidéo ci-dessous.

\section{An interactive or media element has been excluded 只 from this version of the text. You can view it online} here:

https://openeducationalberta.ca/ ressourceseducationcsj/? $p=237$

Maintenant, ci-dessous sélectionnez les 4 éléments utilisez dans la recherche de la vidéo précédente. 
An interactive or media element has been excluded from this version of the text. You can view it online here:

https://openeducationalberta.ca/

ressourceseducationcsj/?p=237 


\section{Exercice 7: Encyclopédies}

Quels sont les trois moyens par lesquels il est possible d'avoir accès à l'encyclopédie Universalis Junior?

An interactive or media element has been excluded
from this version of the text. You can view it online here:

https://openeducationalberta.ca/

ressourceseducationcsj/?p=247 


\section{Exercice 8: Ressources d'appui intégrées aux programmes d'études}

Cet exercice vous permet de revoir vos habiletés à trouver un programme d'étude avec le site LearnAlberta.ca et d'y identifier une ressource d'appui pour l'élève.

En vous servant du site de LearnAlberta.ca, veuillez identifier la ressource d'appui suggérée dans le programme d'étude pour Français langue première (cours régulier), 12e année, section C2 (Cuture et identité) qui répond au RAS suivant "s'affirmer en prenant la parole dans des situations diverses de la vie scolaire et sociale."

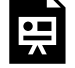

An interactive or media element has been excluded

here:

https://openeducationalberta.ca/

ressourceseducationcsj/? $p=292$

Indice: il est possible d'effectuer une rechercher par mots-clés du RAS sur le texte de la page afin d'identifier rapidement la bonne section. 



\section{PART IV \\ QUESTIONS?}

$\mathrm{Si}$ vous vous posez des questions sur comment trouver des programmes d'étude, des ressources autorisées ou des ressources d'appui, n'hésitez pas à vous adresser au personnel de la bibliothèque par chat ou par e-mail en utilisant le service Question, tel qu'indiqué sur la bannière ci-dessus.

$\mathrm{Si}$ vous souhaitez contacter le bibliothécaire spécialisé en éducation, veuillez trouver ses coordonnées sur le guide de recherche en éducation. 



\section{Glossaire}

\section{Cote}

Il s'agit de l'adresse d'un document qui permet de l'organiser par domaine de recherche ou auteur et de le situer sur les rayons d'une bibliothèque. A la bibliothèque de l'Université de l'Alberta, la cote est d'habitude alphanumérique, par ex. QA 135.6 H8314 2007. En anglais, la cote est connu sous le nom de "call number."

\section{ISBN}

Le Numéro International Normalisé du Livre (ISBN) a 10 ou 13 chiffres uniques pour chaque livre publié. Dans le cas de l'ISBN à 13 chiffres, il commence souvent par les chiffres $978 \ldots$.

\section{Programme d'étude}

Un document produit par le ministère de l'Éducation de l'Alberta qui présente "un ensemble cohérent d'objectifs et d'activités pédagogiques,"(Legendre 1092) ainsi que des résultats d'apprentissage généraux (RAG) et spécifiques (RAS) qui ont été établis dans le Cadre commun.

\section{RAG}

RAG signifie résultats d'apprentissage généraux qui sont des énoncés d'ordre général des principaux apprentissages attendus des élèves dans chacun des domaines ou sousdomaines. Le résultat d'apprentissage général de chaque domaine ou sous-domaine est le même pour tous les niveaux scolaires. (voir la définition dans le programme d'étude Mathématiques $\mathrm{M}$ à 9 , page 10) 


\section{RAS}

RAS signifie résultats d'apprentissage spécifiques qui sont des énoncés qui précisent les habiletés, les connaissances et la compréhension que les élèves devraient avoir acquises à la fin de chaque niveau scolaire.(voir la définition dans le programme d'étude Mathématiques M à 9, page 10)

\section{Vedettes matières}

Les sujets ou vedettes matières sont les grands thèmes représentés dans une ressource. Elles sont standards pour toutes les autres ressources de la bibliothèque du même thème, ce qui facilite la recherche documentaire dans le catalogue. Par exemple, une recherche dans le catalogue de la bibliothèque avec les vedettes matières Mathématiques, étude et enseignement et primaire permettra de trouver toutes les autres ressources de la bibliothèque avec les mêmes vedettes matières et, par conséquent, portant sur l'enseignement des mathématiques au niveau primaire. 